\title{
THE SECOND AWARD \\ OF THE BÔCHER MEMORIAL PRIZE
}

The Annual Meeting of 1924 was marked by the second award of the Bôcher Memorial Prize. On recommendation of the Committee, this prize, which was offered for a notable research memoir in volumes 19-24 of the TransAcTions, was divided equally between Eric Temple Bell for a memoir entitled Arithmetical paraphrases, published in volume 22, and Solomon Lefschetz for a memoir entitled On certain numerical invariants of algebraic varieties with applications to Abelian varieties, in the same volume.

The presentation was made at the beginning of the afternoon session on December 30 by President Birkhoff as chairman of the committee. After some personal references to the mathematician whose name this prize commemorates, he gave the following brief resumés of the papers as analyzed by the committee.

"The memoir by Bell is a fundamental contribution to the theory of numbers. That field of mathematics is largely an aggregation of special results and methods. What have been needed are general principles, serving to unify and extend. Such a general principle is given by Bell. This principle may be stated very simply for a special but a typical case. Suppose that by the development of an elliptic or theta function we have found a linear identity between the sines of the angles $a_{i} x+b_{i} y$ for $i=1,2, \cdots$, then if $f(a, b)$ is an arbitrary even function, Bell shows that we have the like linear identity between the $f\left(a_{i}, b_{i}\right)$. Since we are free to choose the even function, we may deduce as many arithmetical facts as we please from the initial identity. Similarly from a linear identity between cosines we derive a corresponding identity for an arbitrary odd function. These are merely the simplest cases of the 
endless varieties of paraphrases, each involving arbitrary functions.

"The memoir by Lefschetz constitutes an important contribution to the theory of algebraic varieties. In the first part of an extensive paper, rich in detail and employing with facility the methods of algebra, geometry, analysis, the notion of algebraic cycles plays a central part. It is shown for example that a double integral of the first kind on an algebraic surface has no periods relative to algebraic cycles, and that Picard's number $\varrho$ is equal to the number of two-cycles without periods, for integrals of the first kind. Here also is found the first rigorous proof of Noether's theorem on complete intersections, and the extension stating that a non-singular variety of $d$ dimensions $(d>3)$, which is itself a complete intersection in space of $r$ dimensions $(r>4)$ contains only hypersurfaces which are themselves complete intersections. The second part of the paper deals with Abelian varieties, and in particular it contains an evaluation of $\varrho$ for such varieties, known previously for the case $p=2$. Many interesting and novel results concerning the relations between the various numerical invariants are found."

Professor Lefschetz, who was present, in acknowledging the honor done him, spoke of the present standing of the American school of mathematics.

The Committee on award appointed by the Council consisted of Professor G. D. Birkhoff, L. E. Dickson and H. S. White.

The third award, to be announced at the Annual Meeting of 1928 , is to be made with reference to the volumes of the Transactions for the years 1923-1927 inclusive.*

R. G. D. Richardson, Secretary.

\footnotetext{
* See this Bulletin, vol. 30 (1924), p. 193.
} 